\title{
Ettore Majorana: His Scientific and Human Personality
}

\section{Erasmo Recami*}

Università statale di Bergamo, Bergamo, Italy; and INFN-Sezione di Milano, Milan, Italy

E-mail: recami@mi.infn.it

\begin{abstract}
This article reports part of the invited talk delivered by the author at the "International Conference on Majorana Legacy" held at the Department of Physics, University of Catania, Catania, Italy, on 5 and 6 Octorber, 2006: Conference organized for celebrating the 100th anniversary of the birth of the Italian theoretical physicist Ettore Majorana; probably the brightest Italian theoretician of the XX century (Enrico Fermi regarded him as the brightest in the world of his time), even if to many people Majorana is still known mainly for his misterious disappearance, in 1938, when he was 31. In this writing we outline the significance of some publications of his, as well as his life: the biographical data being based on letters, documents, testimonies discovered or collected by the author during almost 30 years, and contained in the book by Recami, "Il Caso Majorana: Epistolario, Testimonianze, Documenti" (initially published by Mondadori, Milan, and presently published, in 2002, by Di Renzo Editore, Rome). At last, some information and comments are added with regard to the manuscripts left unpublished by Majorana.
\end{abstract}

International Conference - Ettore Majorana's legacy and the Physics of the XXI century October 5-6 2006

University of Catania, Italy

\footnotetext{
* Speaker.
} 


\section{Historical Prelude.}

Ettore Majorana's fame solidly rests on testimonies like the following, from the evocative pen of Giuseppe Cocconi. At the request of Edoardo Amaldi[3], he wrote from CERN (July 18, 1965):

"In January 1938, after having just graduated, I was invited, essentially by you, to come to the Institute of Physics at the University in Rome for six months as a teaching assistant, and once I was there I would have the good fortune of joining Fermi, Bernardini (who had been given a chair at Camerino a few months earlier) and Ageno (he, too, a new graduate), in the research of the products of disintegration of $\mu$ "mesons" (at that time called mesotrons or yukons), which are produced by cosmic rays [...]

"It was actually while I was staying with Fermi in the small laboratory on the second floor, absorbed in our work, with Fermi working with a piece of Wilson's chamber (which would help to reveal mesons at the end of their range) on a lathe and me constructing a jalopey for the illumination of the chamber, using the flash produced by the explosion of an aluminum ribbon shortcircuited on a battery, that Ettore Majorana came in search of Fermi. I was introduced to him and we exchanged few words. A dark face. And that was it. An easily forgettable experience if, after a few weeks while I was still with Fermi in that same workshop, news of Ettore Majorana's disappearance in Naples had not arrived. I remember that Fermi busied himself with telephoning around until, after some days, he had the impression that Ettore would never be found.

"It was then that Fermi, trying to make me understand the significance of this loss, expressed himself in quite a peculiar way; he who was so objectively harsh when judging people. And so, at this point, I would like to repeat his words, just as I can still hear them ringing in my memory: 'Because, you see, in the world there are various categories of scientists: people of a secondary or tertiary standing, who do their best but do not go very far. There are also those of high standing, who come to discoveries of great importance, fundamental for the development of science' (and here I had the impression that he placed himself in that category). 'But then there are geniuses like Galileo and Newton. Well, Ettore was one of them. Majorana had what no one else in the world had $[\ldots]$..."

And, with first-hand knowledge, Bruno Pontecorvo, adds: "Some time after his entry into Fermi's group, Majorana already possessed such an erudition and had reached such a high level of comprehension of physics that he was able to speak on the same level with Fermi about scientific problems. Fermi himself held him to be the greatest theoretical physicist of our time. He often was astounded [...]. I remember exactly these words that Fermi spoke: 'If a problem has already been proposed, no one in the world can resolve it better than Majorana.' " (See also [12].)

Ettore Majorana disappeared rather mysteriously on March 26, 1938, and was never seen again [4]. The myth of his "disappearance" has contributed to nothing more than the notoriety he was entitled to, for being a true genius and a genius well ahead of his time.

Majorana was such a pioneer, that even his manuscripts known as the Volumetti, which comprise his study notes written in Rome between 1927, when he abandoned his studies in engineering to take up physics, and 1931, are a paragon not only of order, based on argument and even supplied with an index, but also of conciseness, essentiality and originality: So much so that those notebooks could be regarded as an excellent modern text of theoretical physics, even after about eighty years, and a "gold-mine" of seminal new theoretical, physical, and mathematical ideas and hints, 
quite stimulating and useful for modern research. Such scientific manuscripts, incidentally, have been published for the first time (in 2003) by Kluwer[6]; and republished in 2006 in the original Italian language[7]. But Majorana's most interesting notebooks or papers -those that constituted his "research notes" will not see the light in the near future: it being too hard the task of selecting, interpreting and...electronically typing them! Each notebook was written during a period of about one year, starting from the years - as we said above_ during which Ettore Majorana was completing his studies at the University of Rome. Thus the contents of these notebooks range from typical topics covered in academic courses to topics at the frontiers of research. Despite this unevenness in the level of sophistication, the style in which any particular topic is treated is never obvious. As an example, we refer here to Majorana's study of the shift in the melting point of a substance when it is placed in a magnetic field or, more interestingly, his examination of heat propagation using the "cricket simile." Also remarkable is his treatment of contemporary physics topics in an original and lucid manner, such as Fermi's explanation of the electromagnetic mass of the electron, the Dirac equation with its applications, and the Lorentz group, revealing in some cases the literature preferred by him. As far as frontier research arguments are concerned, let us here recall only two illuminating examples: the study of quasi-stationary states, anticipating Fano's theory by about 20 years, and Fermi's theory of atoms, reporting analytic solutions of the Thomas-Fermi equation with appropriate boundary conditions in terms of simple quadratures, which to our knowledge were still lacking.

Let us recall that Majorana, after having switched to physics at the beginning of 1928, graduated with Fermi on July 6, 1929, and went on to collaborate with the famous group created by Enrico Fermi and Franco Rasetti (at the start with O.M.Corbino's important help); a theoretical subdivision of which was formed mainly (in the order of their entrance into the Institute) by Ettore Majorana, Gian Carlo Wick, Giulio Racah, Giovanni Gentile Jr., Ugo Fano, Bruno Ferretti, and Piero Caldirola. The members of the experimental subgroup were: Emilio Segré, Edoardo Amaldi, Bruno Pontecorvo, Eugenio Fubini, Mario Ageno, Giuseppe Cocconi, along with the chemist Oscar D'Agostino. Afterwards, Majorana qualified for university teaching of theoretical physics ("Libera Docenza") on November 12, 1932; spent about six months in Leipzig with W. Heisenberg during 1933; and then, for some unknown reasons, stopped participating in the activities of Fermi's group. He even ceased publishing the results of his research, except for his paper "Teoria simmetrica dell'elettrone e del positrone," which (ready since 1933) Majorana was persuaded by his colleagues to remove from a drawer and publish just prior to the 1937 Italian national competition for three full-professorships.

With respect to the last point, let us recall that in 1937 there were numerous Italian competitors for these posts, and many of them were of exceptional caliber; above all: Ettore Majorana, Giulio Racah, Gian Carlo Wick, and Giovanni Gentile Jr. (the son of the famous philosopher bearing the same name, and the inventor of "parastatistics" in quantum mechanics). The judging committee was chaired by E. Fermi and had as members E. Persico, G. Polvani, A. Carrelli, and O. Lazzarino. On the recommendation of the judging committee, the Italian Minister of National Education installed Majorana as professor of theoretical physics at Naples University because of his "great and well-deserved fame," independently of the competition itself; actually, "the Commission hesitated to apply the normal university competition procedures to him." The attached report on the scientific activities of Ettore Majorana, sent to the minister by the committee, stated: 
"Without listing his works, all of which are highly notable both for their originality of the methods utilized as well as for the importance of the achieved results, we limit ourselves to the following:

"In modern nuclear theories, the contribution made by this researcher to the introduction of the forces called "Majorana forces" is universally recognized as the one, among the most fundamental, that permits us to theoretically comprehend the reasons for nuclear stability. The work of Majorana today serves as a basis for the most important research in this field.

"In atomic physics, the merit of having resolved some of the most intricate questions on the structure of spectra through simple and elegant considerations of symmetry is due to Majorana.

"Lastly, he devised a brilliant method that permits us to treat the positive and negative electron in a symmetrical way, finally eliminating the necessity to rely on the extremely artificial and unsatisfactory hypothesis of an infinitely large electrical charge diffused in space, a question that had been tackled in vain by many other scholars."

One of the most important works of Ettore Majorana, the one that introduces his "infinitecomponents equation" was not mentioned, since it had not yet been understood. It is interesting to note, however, that the proper light was shed on his theory of electron and anti-electron symmetry (today climaxing in its application to neutrinos and anti-neutrinos) and on his resulting ability to eliminate the hypothesis known as the "Dirac sea," a hypothesis that was defined as "extremely artificial and unsatisfactory," despite the fact that in general it had been uncritically accepted.

The details of Majorana and Fermi's first meeting were narrated by E. Segré [14]: "The first important work written by Fermi in Rome ['Su alcune proprietà statistiche dell'atomo' (On certain statistical properties of the atom)] is today known as the Thomas-Fermi method.... When Fermi found that he needed the solution to a non-linear differential equation characterized by unusual boundary conditions in order to proceed, in a week of assiduous work with his usual energy, he calculated the solution with a little hand calculator. Majorana, who had entered the Institute just a short time earlier and who was always very skeptical, decided that Fermi's numeric solution probably was wrong and that it would have been better to verify it. He went home, transformed Fermi's original equation into a Riccati equation, and resolved it without the aid of any calculator, utilizing his extraordinary aptitude for numeric calculation. When he returned to the Institute and skeptically compared the little piece of paper on which he had written his results to Fermi's notebook, and found that their results coincided exactly, he could not hide his amazement." We have indulged in the foregoing anecdote since the pages on which Majorana solved Fermi's differential equation have in the end been found, and it has been shown recently [11] that he actually followed two independent (and quite original) paths to the same mathematical result, one of them leading to an Abel, rather than a Riccati, equation.

Majorana delivered his lectures only during the beginning of 1938, starting on Jan.13 and ending with his disappearance (March 26). But his activity was intense, and his interest for teaching extremely high. For the benefit of his beloved students, and perhaps also for writing down a book, he prepared careful notes for his lectures. And ten of such lectures appeared in print in 1987 (see ref.[1]): and arose the admired comments of many (especially British) scholars. The remaining six lecture-notes, which had gone lost, have been rediscovered in 2005 by Salvatore Esposito and Antonino Drago, and just appeared in print[2]. 


\section{Ettore Majorana's Published Papers.}

Majorana published few scientific articles: nine, actually, besides his sociology paper entitled "Il valore delle leggi statistiche nella fisica e nelle scienze sociali" (The value of statistical laws in physics and the social sciences), which was however published not by Majorana but (posthumously) by G. Gentile Jr., in Scientia [36 (1942) 55-56]. We already know that Majorana switched from engineering to physics in 1928 (the year in which he published his first article, written in collaboration with his friend Gentile) and then went on to publish his works in theoretical physics only for a very few years, practically only until 1933. Nevertheless, even his published works are a mine of ideas and techniques of theoretical physics that still remains partially unexplored. Let us list his nine published articles:

(1) "Sullo sdoppiamento dei termini Roentgen ottici a causa dell'elettrone rotante e sulla intensità delle righe del Cesio," in collaboration with Giovanni Gentile Jr., Rendiconti Accademia Lincei 8 (1928) 229-233.

(2) "Sulla formazione dello ione molecolare di He," Nuovo Cimento 8 (1931) 22-28.

(3) "I presunti termini anomali dell'Elio," Nuovo Cimento 8 (1931) 78-83.

(4) "Reazione pseudopolare fra atomi di Idrogeno," Rendiconti Accademia Lincei 13 (1931) 5861 .

(5) “Teoria dei tripletti $P$ ' incompleti," Nuovo Cimento 8 (1931) 107-113.

(6) "Atomi orientati in campo magnetico variabile," Nuovo Cimento 9 (1932) 43-50.

(7) "Teoria relativistica di particelle con momento intrinseco arbitrario," Nuovo Cimento 9 (1932) 335-344.

(8) "Über die Kerntheorie," Zeitschrift für Physik 82 (1933) 137-145; and "Sulla teoria dei nuclei," La Ricerca Scientifica 4(1) (1933) 559-565.

(9) “Teoria simmetrica dell'elettrone e del positrone," Nuovo Cimento 14 (1937) 171-184.

The first papers, written between 1928 and 1931, concern atomic and molecular physics: mainly questions of atomic spectroscopy or chemical bonds (within quantum mechanics, of course). As E. Amaldi has written [3], an in-depth examination of these works leaves one struck by their superb quality: They reveal both a deep knowledge of the experimental data, even in the minutest detail, and an uncommon ease, without equal at that time, in the use of the symmetry properties of the quantum states in order to qualitatively simplify problems and choose the most suitable method for their quantitative resolution. Among the first papers, "Atomi orientati in campo magnetico variabile" (Atoms oriented in a variable magnetic field) deserves special mention. It is in this article, famous among atomic physicists, that the effect now known as the Majorana-Brossel effect is introduced. In it, Majorana predicts and calculates the modification of the spectral line shape due to an oscillating magnetic field. This work has also remained a classic in the treatment of non-adiabatic spin-flip. Its results —once generalized, as suggested by Majorana himself, by Rabi in 1937 and 
by Bloch and Rabi in 1945 - established the theoretical basis for the experimental method used to reverse the spin also of neutrons by a radio-frequency field, a method that is still practiced today, for example, in all polarized-neutron spectrometers. This Majorana paper does moreover introduce the so-called Majorana sphere (to represent complex spinors by a set of three, or two, points on the surface of a sphere), as noted not long ago by R.Penrose[15] and others[16].

Majorana's last three articles are all of such importance that none of them can be set aside without comment.

The article "Teoria relativistica di particelle con momento intrinseco arbitrario" (Relativistic theory of particles with arbitrary spin) is a typical example of a work that is so far ahead of its time that it became understood and evaluated in depth only many years later. Around 1932 it was commonly thought that one could write relativistic quantum equations only in the case of particles with zero or half spin. Convinced of the contrary, Majorana - as we know from his manuscriptsbegan constructing suitable quantum-relativistic equations [10] for higher spin values (one, threehalves, etc.); and he even devised a method for writing the equation for a generic spin-value. But still he published nothing, until he discovered that one could write a single equation to cover an infinite series of cases, that is, an entire infinite family of particles of arbitrary spin (even if at that time the known particles could be counted on one hand). In order to implement his programme with these "infinite components" equations, Majorana invented a technique for the representation of a group several years before Eugene Wigner did. And, what is more, Majorana obtained the infinitedimensional unitary representations of the Lorentz group that will be re-discovered by Wigner in his 1939 and 1948 works. The entire theory was re-invented by Soviet mathematicians (in particular Gelfand and collaborators) in a series of articles from 1948 to 1958 and finally applied by physicists years later. Sadly, Majorana's initial article remained in the shadows for a good 34 years until D. Fradkin, informed by E. Amaldi, released [Am. J. Phys. 34 (1966) 314] what Majorana many years earlier had accomplished.

As soon as the news of the Joliot-Curie experiments reached Rome at the beginning of 1932, Majorana understood that they had discovered the "neutral proton" without having realized it. Thus, even before the official announcement of the discovery of the neutron, made soon afterwards by Chadwick, Majorana was able to explain the structure and stability of atomic nuclei with the help of protons and neutrons, antedating in this way also the pioneering work of D. Ivanenko, as both Segré and Amaldi have recounted. Majorana's colleagues remember that even before Easter he had concluded that protons and neutrons (indistinguishable with respect to the nuclear interaction) were bound by the "exchange forces" originating from the exchange of their spatial positions alone (and not also of their spins, as Heisenberg would propose), so as to produce the alpha particle (and not the deuteron) saturated with respect to the binding energy. Only after Heisenberg had published his own article on the same problem was Fermi able to persuade Majorana to meet his famous colleague in Leipzig; and finally Heisenberg was able to convince Majorana to publish his results in the paper "Über die Kerntheorie." Majorana's paper on the stability of nuclei was immediately recognized by the scientific community -a rare event, as we know, from his writings- thanks to that timely "propaganda" made by Heisenberg himself. We seize the present opportunity to quote two brief passages from Majorana's letters from Leipzig. On February 14, 1933, he writes his mother (the italics are ours): "The environment of the physics institute is very nice. I have good relations with Heisenberg, with Hund, and with everyone else. I am writing some articles in German. The 
first one is already ready...." The work that is already ready is, naturally, the cited one on nuclear forces, which, however, remained the only paper in German. Again, in a letter dated February 18, he tells his father (we italicize): "I will publish in German, after having extended it, also my latest article which appeared in Nuovo Cimento." Actually, Majorana published nothing more, either in Germany or after his return to Italy, except for the article (in 1937) of which we are about to speak. It is therefore of importance to know that Majorana was engaged in writing other papers: in particular, he was expanding his article about the infinite-components equations.

As we said, from the existing manuscripts it appears that Majorana was also formulating the essential lines of his symmetric theory of electrons and anti-electrons during the years 1932-1933, even though he published this theory only years later, when participating in the forementioned competition for a professorship, under the title "Teoria simmetrica dell'elettrone e del positrone" (Symmetrical theory of the electron and positron), a publication that was initially noted almost exclusively for having introduced the Majorana representation of the Dirac matrices in real form. A consequence of this theory is that a neutral fermion has to be identical with its anti-particle, and Majorana suggested that neutrinos could be particles of this type. As with Majorana's other writings, this article also started to gain prominence only decades later, beginning in 1957; and nowadays expressions like Majorana spinors, Majorana mass, and Majorana neutrinos are fashionable. As already mentioned, Majorana's publications (still little known, despite it all) is a potential gold-mine for physics. Many years ago, for example, Bruno Touschek noticed[8] that the present article implicitly contains also what he called the theory of the "Majorana oscillator", described by the simple equation $q+\omega^{2} q=\varepsilon \delta(t)$, where $\varepsilon$ is a constant and $\delta$ the Dirac function[9]. According to Touschek, the properties of the Majorana oscillator are very interesting, especially in connection with its energy spectrum: But no literature seems to exist yet on it. Another example: much more recently, C.Becchi pointed out how, in the first pages of the present paper, a clear formulation of the quantum action principle appears, the same principle that in later years, through Schwinger's and Symanzik's works, for example, has brought about quite important advances in quantum field theory.

\section{Ettore Majorana's Unpublished Papers.}

Majorana also left us several unpublished scientific manuscripts, all of which have been catalogued [10] and kept at the "Domus Galilaeana" of Pisa, Italy. Our analysis of these manuscripts has allowed us to ascertain that all the existing material seems to have been written by 1933; even the rough copy of his last article, which Majorana proceeded to publish in 1937 —as already mentioned - seems to have been ready by 1933 , the year in which the discovery of the positron was confirmed. Indeed, we are unaware of what he did in the following years from 1934 to 1938 , except for a series of 34 letters written by Majorana between March 17, 1931, and November 16, 1937, in reply to his uncle Quirino - a renowned experimental physicist and at a time president of the Italian Physical Society_ who had been pressing Majorana for theoretical explanations of his own experiments. By contrast, his sister Maria recalled that, even in those years, Majorana - who had reduced his visits to Fermi's Institute, starting from the beginning of 1934 (that is, after his return from Leipzig) — continued to study and work at home many hours during the day and at night. Did he continue to dedicate himself to physics? From a letter of his to Quirino, dated January 16, 
1936, we find a first answer, because we get to learn that Majorana had been occupied "since some time, with quantum electrodynamics"; knowing Majorana's modesty and love for understatements, this no doubt means that by 1935 Majorana had profoundly dedicated himself to original research in the field of quantum electrodynamics.

Do any other unpublished scientific manuscripts of Majorana exist? The question, raised by his letters from Leipzig to his family, becomes of greater importance when one reads also his letters addressed to the National Research Council of Italy (CNR) during that period. In the first one (dated January 21, 1933), Majorana asserts: "At the moment, I am occupied with the elaboration of a theory for the description of arbitrary-spin particles that I began in Italy and of which I gave a summary notice in Nuovo Cimento...." In the second one (dated March 3, 1933) he even declares, referring to the same work: "I have sent an article on nuclear theory to Zeitschrift für Physik. I have the manuscript of a new theory on elementary particles ready, and will send it to the same journal in a few days." Considering that the article described here as a "summary notice" of a new theory was already of a very high level, one can imagine how interesting it would be to discover a copy of its final version, which went unpublished. [Is it still, perhaps, in the Zeitschrift für Physik archives? Our own search ended in failure]. One must moreover not forget that the abovecited letter to Quirino Majorana, dated January 16, 1936, revealed that his nephew continued to work on theoretical physics even subsequently, occupying himself in depth, at least, with quantum electrodynamics.

Some of Majorana's other ideas, when they did not remain concealed in his own mind, have survived in the memories of his colleagues. One such reminiscence we owe to Gian Carlo Wick. Writing from Pisa on October 16, 1978, he recalls: “...The scientific contact [between Ettore and me], mentioned by Segré, happened in Rome on the occasion of the 'A. Volta Congress' (long before Majorana's sojourn in Leipzig). The conversation took place in Heitler's company at a restaurant, and therefore without a blackboard...; but even in the absence of details, what Majorana described in words was a 'relativistic theory of charged particles of zero spin based on the idea of field quantization' (second quantization). When much later I saw Pauli and Weisskopf's article [Helv. Phys. Acta 7 (1934) 709], I remained absolutely convinced that what Majorana had discussed was the same thing...."

We attach to this paper a short Bibliography. Far from being exhaustive, it provides only some references about the topics touched upon in this paper.

\section{Acknowledgments.}

The author is quite grateful to all the Organizers of this Catania Conference: A. Agodi, P. Castorina, F. Catara, E. Migneco, S. Lo Nigro, F. Porto, E. Rimini, and in particular A. Rapisarda. Moreover, for constant encouragement or collaboration, he thanks Franco Bassani, Salvatore Esposito, Ignazio Licata, Fabio Majorana, Bruno Preziosi and Silvano Sgrignoli.

\section{References}

[1] Ettore Majorana - Lezioni all'Università di Napoli, edited by B.Preziosi (Bibliopolis pub.; Neaples, 1987). 
[2] Lezioni di Fisica Teorica - Ettore Majorana, edited by S.Esposito (Bibliopolis pub.; Neaples, 2006).

[3] E.Amaldi, La Vita e l'Opera di E. Majorana (Accademia dei Lincei; Roma, 1966); "Ettore Majorana: Man and scientist," in Strong and Weak Interactions. Present problems, edited by A.Zichichi (Academic Press; New York, 1966); "Ricordo di Ettore Majorana", in Giornale di Fisica 9 (Bologna, 1968) p.300; E. Amaldi: "From the discovery of the neutron to the discovery of nuclear fission", in Physics Reports 111 (1984) pp.1-322; E. Amaldi: in Il Nuovo Saggiatore 4 (Bologna, 1988) p.13.

[4] The documents that appear in this article (as well as all the biographic documentation regarding E.Majorana, directly discovered or collected by E.Recami in almost 30 years) can be found in the book by E.Recami: Il caso Majorana: Epistolario, Documenti, Testimonianze, 2nd edition (Oscar, Mondadori pub.; Milano, 1991), pp.230; and in particular in its 4th edition (Di Renzo pub.; Roma, 2002), pp.273. [They are protected since 1986 by copyright in favour of Maria Majorana together with E.Recami, and, now, with Di Renzo Publisher]. Of those book it exists a good translation into French, performed by F. and $\mathrm{Ph}$. Gueret (unpublished).

[5] See also E. Recami: "I nuovi documenti sulla scomparsa di E.Majorana", in Scientia 110 (1975) p.577; in La Stampa (Turin), 1st June and 29 June 1975; in Corriere della Sera (Milan), 19 October 1982 and 13 December 1983; "Ricordo di Ettore Majorana a sessant' anni dalla sua scomparsa: L'opera scientifica edita e inedita", in Quaderni di Storia della Fisica (S.I.F.), 5 (1999), pp.19-68; and moreover VV.AA.: Scienziati e tecnologi contemporanei: Enciclopedia Biografica, 3 vols., edited by E.Macorini (Mondadori pub.; Milan, 1974); M.Farinella: in L'Ora (Palermo), 22 and 23 July 1975; G.C.Graziosi: "Le lettere del mistero Majorana", in Domenica del Corriere (Milan), 28 November 1972; S.Ponz de Leon: "Speciale News: Majorana", TV piece broadcast on 30 September 1987 ("Canale Cinque"); B.Russo: "Ettore Majorana - Un giorno di marzo", TV piece broadcast on 18 December 1990 ("RAI Tre-Sicilia"); F.Tomandl e A.Stadler: large radio piece on Ettore Majorana broadcast at the end of 2004 by the "Austrian Radio", Vienna.

[6] Ettore Majorana - Notes on Theoretical Physics, edited by S.Esposito, E.Majorana Jr., A. van der Merwe, and E.Recami (Kluwer Acad. Pub.; Dordrecht, Boston and London, Nov. 2003).

[7] Ettore Majorana - Appunti Inediti di Fisica Teorica, edited by S.Esposito and E.Recami (Zanichelli pub.; Bologna, 2006).

[8] B.Touschek: private communications (1976). Thanks are due to E.Pessa for having forwarded to us, at that time, Touschek's comment.

[9] See page 70 in the IV edition (2002) of the book by Recami, quoted in ref.[4].

[10] M.Baldo, R.Mignani e E.Recami: "About a Dirac-like equation for the photon, according to Ettore Majorana”, Lett. Nuovo Cimento 11 (1974) 568 [interesting also for a possible physical interpretation of the photon wave-function]. See also S.Esposito: Found. Phys. 28 (1998) 231; and E.Giannetto; Lett. Nuovo Cimento 44 (1985) 140 e 145; "Su alcuni manoscritti inediti di E.Majorana", in Atti IX Congresso Naz.le di Storia della Fisica, edited by F.Bevilacqua (Milan, 1988) p.173.

[11] S.Esposito: "Majorana solution of the Thomas-Fermi equation", Am. J. Phys. 70 (2002) 852-856; "Majorana transformation for differential equations", Int. J. Theor. Phys. 41 (2002) 2417-2426.

[12] B.Pontecorvo, Fermi e la fisica moderna (Editori Riuniti pub.; Rome, 1972); and in Proceedings International Conference on the History of Particle Physics, Paris, July 1982, Physique 43 (1982).

[13] Cf. also, e.g., G.Enriques: Via D’Azeglio 57 (Zanichelli pub.; Bologna, 1971).

[14] E.Segré: Enrico Fermi, Fisico (Zanichelli; Bologna (1971); and Autobiografia di un Fisico (Il Mulino pub.; Rome, 1995). 
[15] R.Penrose, "Newton, quantum theory and reality," in 300 Years of Gravitation, edited by S.W.Hawking and W.Israel (University Press; Cambridge, 1987); J. Zimba and R. Penrose, Stud. Hist. Phil. Sci. 24 (1993) 697; R. Penrose: Ombre della Mente (Shadows of the Mind) (Rizzoli pub.; 1996), pp.338-343 and 371-375; and the subsequent studies, performed at Palermo, by C.Leonardi, F.Lillo, A.Vaglica e G.Vetri: “ Quantum visibility, phase-difference operators, and the Majorana Sphere", preprint (Phys.Dept., Univ. of Palermo, Italy; 1998); "Majorana and Fano alternatives to the Hilbert space", in Mysteries, Puzzles, and Paradoxes in Quantum Mechanics, ed. by R.Bonifacio (A.I.P.; Woodbury, N.Y., 1999), pp.312-315; F.Lillo: “Aspetti Fondamentali nell'Interferometria a Uno e Due Fotoni", PhD thesis (C.Leonardi supervisor), Dept. of Physics, University of Palermo, 1998.

[16] Majorana Legacy in Contemporary Physics, edited by I.Licata (Di Renzo pub.; Rome, 2006), which can be found also in the electronic journal EJTP Electr. J. Theor. Phys. 3 (2006), issue no.10.

[17] M.Baldo, R.Mignani, e E.Recami, "Catalogo dei manoscritti scientifici inediti di E. Majorana," in Ettore Majorana - Lezioni all'Università di Napoli, edited by B.Preziosi (Bibliopolis pub.; Napoli, 1987), pp.175-197; and E. Recami, "Ettore Majorana: L'opera edita ed inedita," Quaderni di Storia della Fisica (of the Giornale di Fisica) (S.I.F. pub.; Bologna) 5 (1999) 19-68. 